METEOROLOGICAL REGISTERS

THERE is now scarcely a meteorological observatory which is not provided with registering instruments. The number of these is already considerable,

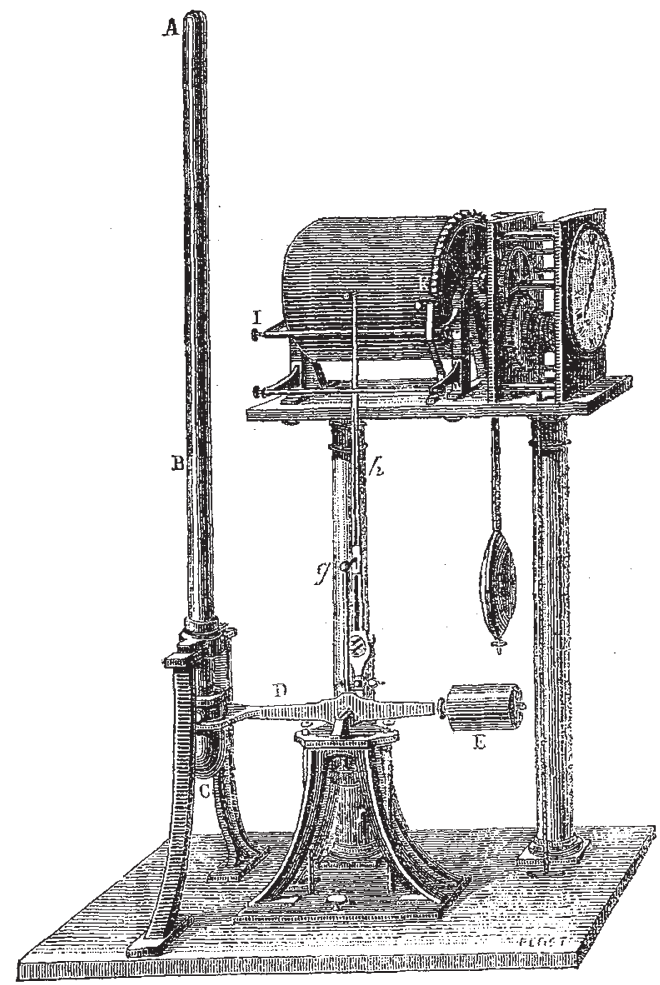

Frg. I.-Registering Barometer. plunger being equal to that which corresponds in the tube to the increase in height of the barometric column) the level of mercury in the cistern returns to its constant point, and when, consequently, the apparent weight of that cistern has resumed its fixed value. The needle, $h$, of the beam of the balance marks its displacements on the surface of the blackened cylinder, $\mathrm{K}$. It only remains to read the indications. To do this as accurately as possible, the support of the cylinder carries an electro-magnet, the armature of which is provided with an arm bearing a metallic point. ${ }^{1}$ In ordinary weather this point is fixed, and traces on the cylinder a datum-line from which the ordinates of the barometric curve are measured; but every hour the electro-magnet is acted on by a clock marking the time on all the registers; the point departs momentarily from the line, and makes a clear stroke, which is reproduced at the same instant on the other cylinders. At the end of each week the cylinder is taken away, to be replaced by another kept ready, and is placed on the reading apparatus.

This apparatus (Fig. 2 ) consists of a horizontal steel bench, $f g$; provided on its upper surface with two columns, $h h^{\prime}$, intended to support the axis of the cylinder, and on a vertical stand with two microscopes, which may be moved either separately or together in a horizontal direction. By means of an endless screw a movement of rotation on its axis may be communicated to the cylinder for the purpose of presenting successively to the microscopes the various faces of the cylinder. The microscope $b$ is always pointed on the datum-line, and it is to follow this line in its accidental displacements, that the two microscopes may be moved together by the action of an adjusting screw. The microscope which points to the traced line carries at its focus two crossed threads, one horizontal and the other vertical. At each hourly reading one of the horizontal tracings on the datum-line is brought under the horizontal thread of the microscope. But, as the barometric curve often presents inflexions of Whieh it is useful to note the exact time, the critical point of this curve being brought under the movable microscope $a$, a second horizontal thread, movable by a micrometric screw, serves to measure the distance of this point from the hourly mark immediately preceding.

and the methods of their construction are almost as numerous. At the observatory of Montsouris I have preferred continuous registration to registration by points, and the tracings effected by a fine metallic point on glazed paper blackened with smoke to the use of the pencil or to the ganfrage by pressure or by shock:

The barograph of Montsouris is a barometer-balance devised by P. Cecci, and latterly adopted by $P$. Secchi. The barometric tube A B (Fig. $x$ ) is of iron, of 3 centimetres internal diameter, and of the same calibre throughout its length. It is fixed, and has, at its lower extremity, a cylindrical plunger, the full section of which is equal to the vacuum section of the barometric tube. The cistern, $\mathrm{C}$, is of steel, and suspended to one end of the beam of a balance, $D$, the other end of which carries a counterpoise. The centre of gravity of the weighted beam coincides, as nearly as possible, with the central knife-edge, so as to render the equilibrium indifferent. Hence it results that when, in consequence of a rise of the barometer, a part of the mercury rises from the cistern into the tube, the former, thus rendered lighter, tends to rise, with the effect of introducing

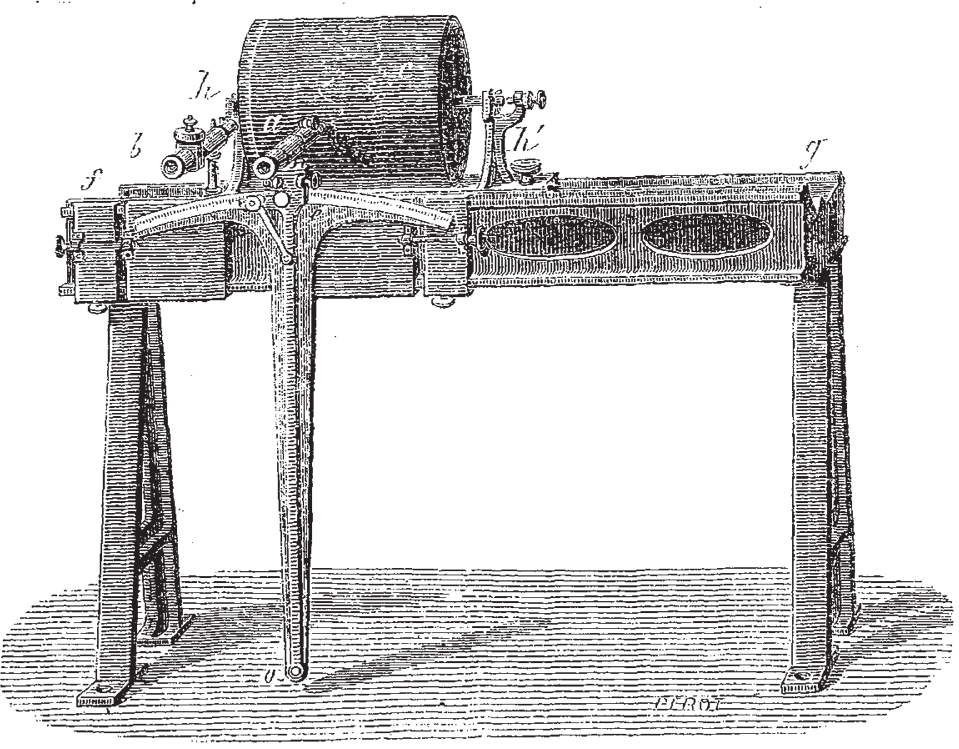

FiG. 2.-Micrometer or reading curves traced by. the register.

into the mercury of the cistern a greater length of The microscope $a$ is supported at the extremity of an the cylindrical plunger. Equilibrium is only re-estab- arm of a lever the length of which is equal to that of the lished when (the volume of mercury displaced by the ${ }^{x}$ This electro-magnet, which is iseen in ithe thermcgraph ( $F \cdot 3$. 3 ), is not x Paper by M. Marié-Davy, Yournal de Phy'sigte, April, r87g. 
barometric needle; it describes, consecuently, an arc of a circle of the same radius as the point of the register. This second microscope has a cross of threads like the first, but without a movable thread. The first microscope being pointed to an hourly sign, the axis of the second is directed to the corresponding point of the curve. The sine of the angle of position of its lever, multiplied by a

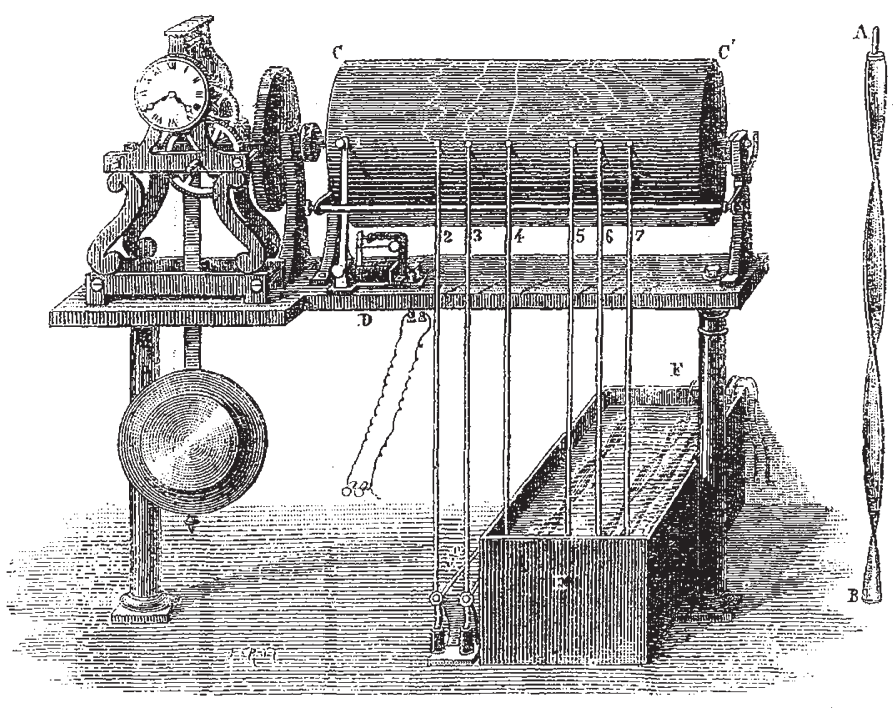

Fig. 3.-Thermograph.

constant factor and increased by a number equally constant, gives the height of the barometer at the moment selected. When the reading of the curve is made and verified, the sheet of blackened paper is taken off the cylinder and soaked in a weak sorution of gurntac or of copal in alcohol. By this means the smoked sheet is "fixed" and thereafter put in a portfolio.

The feeblest barometric variations are thus shown with

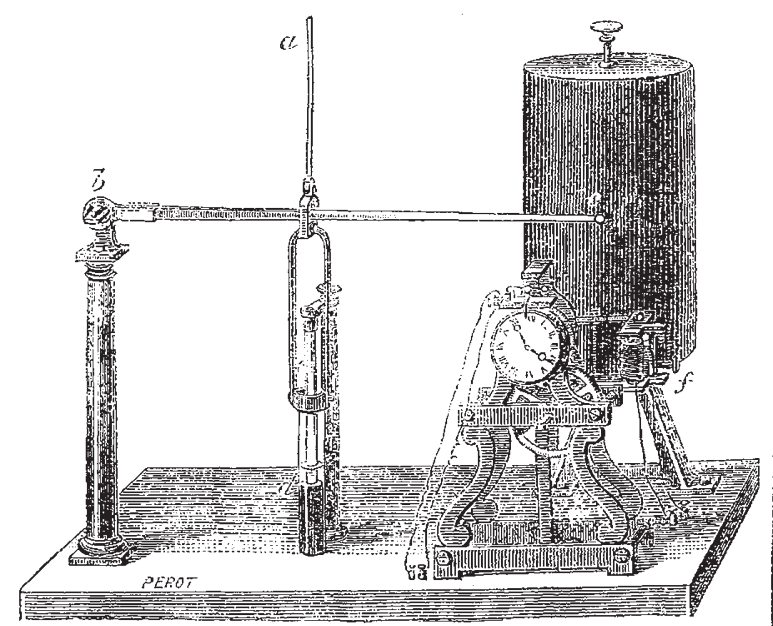

FIG. 4,--Register of the Atmograph.

great fidelity, and by examination of the curves we readily recognise the influence which the dynamical state of the atmosphere exercises in increasing or diminishing the weight of its pressure on the ground. It should be added that the uniformity of the calibre of the barometric tube annuls almost completely the action of temperature on the indications of the instrument.
The thermographs act in an analogous way, except that the motor is here formed by a Bourdon tube of hammerhardened copper, with a very elongated elliptical section, and twisted into a sort of spiral, the thread of the spire varying from 2 to 3 centimetres, according to the purpose for which it is intended. A B (Fig. 3) represents one of these tubes, forming a little more than one spiral. This tube is exactly filled with alcohol, and closed at its two ends. The expansion of the alcohol compels it to untwist; but in order that its elasticity may be preserved, the internal pressure, corresponding to the highest temperatures to be reached, should not exceed 8 or 9 atmospheres. This pressure is all the greater in proportion as the thread of the spire is short. The twisted tube is fixed by one of its ends, the other free extremity bears the needle which gives the indications. At Montsouris this needle is 5 centimetres long, as is also that of the barometer. The process of reading the curves is thus exactly the same for all the needle registers. The new thermograph of Montsouris Observatory contains seven needles acting on the same cylinder. The first needle (No. I, Fig. 3) belongs to the electro-magnet which traces the datum-line, and the hourly signs; needles 2 and 3 belong to the psychrometer; needles 4 and 5 record the temperature of the surface of ground exposed to the air without shelter; needles 6 and 7 correspond to the actinometer.

The psychrometer is formed of two twisted tubes placed outside on the north face of the wooden kiosk, which shelters the cylinder, and perpendicular to that face. Their furthest extremity is fixed, the other is prolonged through the wall of the kiosk by a stem of copper carrying the indicating needle. One of the twisted tubes is uncovered, and forms the dry thermometer; the other is covered with cambric, and kept moist by means of cotton-wicks dipped in small glass cups, connected with a Mariotte flask placed in the kiosk by means of a long and fine tube of caoutchouc. An instrument of this kind has been in use for fifteen months at Montsouris; its action is regular, and its sensibility very great.

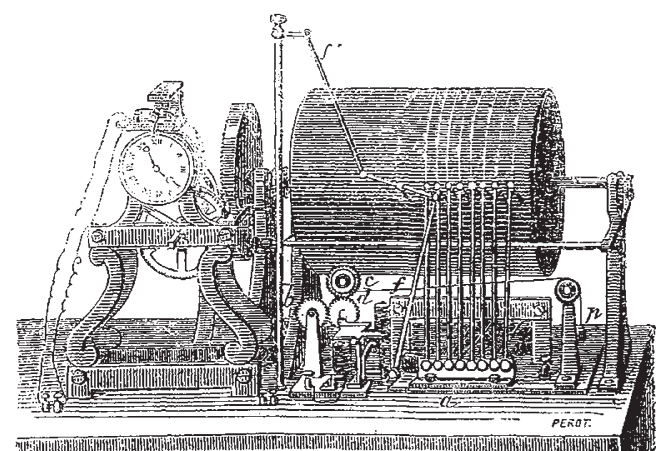

FIG. 5.-Anemograph.

The earth-thermometer is composed of two parts:-A thermometric reservoir of black copper is placed on the surface of a mass of vegetable mould, the top of which is fush with the platform of the roof of the kiosk; this reservoir communicates by a capillary tube of copper with a twisted tube placed in the kiosk under the cylinder of the register. When the temperature of the ground rises, a portion of the alcohol passes from the upper reservoir into the twisted tube, the pressure increases and the tube untwists; but the twisted tube itself and its capillary tube of 
copper, are themselves acted on by the variable temperatures which complicate the results. A second twisted tube, similar to the first, provided with its capillary tube, serves to give the necessary corrections. The tro twisted tubes are arranged parallel to each other in a trough, EF, filled with glycerinated water; the two capillary tubej are soldered to each other throughout their length. The two needles trace two curves, one very siightly sinuous, the other very undulating; the difierence of their ordinates is measured. We thus obtain the temperatures of ground exposed to the sun during day, to nocturnal radiation during night, to rain and to evaporation.

The actinometer is also composed of two twisted tubes resting parailel to each other in the above-mentioned trough, E F, and of two capillary tubes. To each of these tubes corresponds a reservoir placed at the top of the roof of the kiost, and inclosed in an envelope of glass on which a dry vacum has been made. One of the reser-

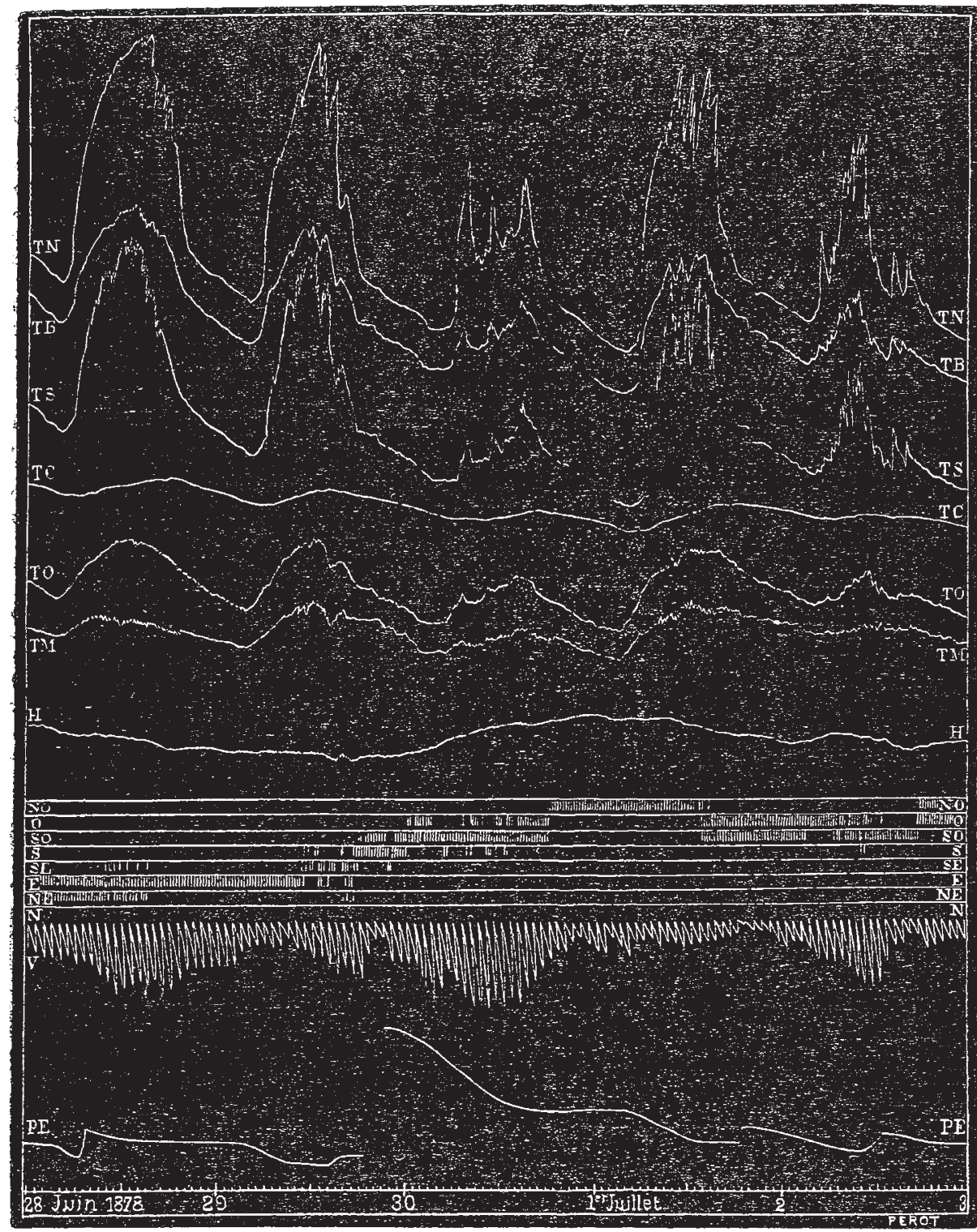

FiG. 6. - Speciman of curyes made by the registers of Montsouris.

woirs is silvered, the other is blackened. Placed symmeerically at a little distance from each other, and far from any shelter, they give the same indications during the night; but during the day the black thermometer registers higher than the white one. The differences of the ordinates of the curves traced by the tivo needles serve to measure the actinic degree. Every cloud which passes over the sun gives rise to a rapid and considerable lowering of the temperatures of the white and black thermometers, and of that on the surface of the ground.

The atmograph is a third registering instrument intended to mark the variations of weight of a mass of earth whose surface is flush with the summit of the platform of the roof of the kiosk, and is exposed to the sun, to evaporation, rain, snow, and dew. At a little distance is the registering rain-gauge. The comparison of the 
two curves shows what becomes of the water which falls on the naked earth, without vegetation, distinguishing between what returns to the atmosphere by evaporation, and what penetrates the subsoil which is permeable or drained. Another atmograph gives similar indications for a soil covered with various plants; but the latter being sheltered from the wind, ought to be moistened according as is necessary. Fig. 4 represents only the register of the atmograph. $a$ is the lower part of the stem which is suspended to the extremity of the arm of the balance, in which is placed the mass of earth. A second lever arm, $b c$, follows and amplifies the movements of that stem which it inscribes on the vertical cylinder covered with paper blackened with smoke. This same stem bears a glass test-tube, $d$, containing mercury, in which is a fixed glass tube $e$. The diameter of this stem is so calculated that the point $c$ traverses 100 millimetres for each millimetre of water gained or lost by the mass of earth. In calm weather we may thus appreciate the ${ }^{2}$ ot $\sigma^{\text {th }}$ of a millimetre; but when the atmosphere is disturbed, the vertical components of the wind cause the needle to oscillate, thus interfering with the precision of the readings.

The anemograph (Fig. 5) gives us, at once, the direction of the wind and its mean rate per hour. Eight electro-magnets communicating electrically with the sectors arranged on the vane according to the eight principal points of the compass, can, by acting singly or two and two, record the winds for sixteen directions, which may be regarded to be sufficient for the wants of meteorology. A ninth electro-magnet is acted on each time that the Robinson drum shows a length of one kilometre traversed by the wind. The toothed wheel $b$ then moves one division, and its movement is transmitted by the satellite wheel $d$ to a third toothed wheel, $c$, on the axis of which is rolled a thread, $c p$. The point which marks the velocity then advances $\mathrm{I} \mathrm{mm}$. towards the left. This effect is produced during one hour for each kilometre traversed by the wind; but at the end of each hour the needle of the clock establishes an electric contact; the satellite wheel, $d$, is lowered; the wheel, $\epsilon$, becomes free; and the weight, $p$, restores the metallic point to its starting-place.

Fig. 6 presents a specimen of the curves traced by the registers from June 28 to July 3,1878 , reduced to onethird of their natural size. Beginning at the top, we find first the two curves, $T \mathrm{~N}$ and $\mathrm{T} B$, which together furnish the actinometric data; $T N$ is the curve of the black thermometer, $\mathrm{T} B$ that of the silvered. The two following curves, $\mathrm{T} S$ and $\mathrm{TC}$, give the temperature of the surface of the ground without shade; TS corresponds to the ground thermometer; $T C$ gives the correction to be made in the ordinates of the first. The two curves, TO and TM, are those of the dry and wet thermometers; besides the temperature of the air in the shade, they give its hygrometric degree and the elastic force of its vapour. $H$ is the curve of barometric pressure.

Underneath are eight straight lines corresponding to the eight principal directions of the wind; the vertical lines which rest on them indicate the directions in which the wind has blown. Further down are shown the velocity, $v$, of the wind in kilometres per hour.

The last curve, $P E$, is made by the atmometer; the increase in a vertical direction of this curve marks rain; the decrease marks evaporation. Notwithstanding the frequent and at one time very copious rains, the earth, on July 3 , had very nearly resumed its weight of June 28 . Finally, the last line is the datum-line on which the hours of the day are marked.

\section{GERMAN PHYSIOLOGICAL CHEMISTRY}

$\triangle S$ our general knowledge of nature increases, the possibility of individual knowledge decreases; the variety of discovery, the immense number of investix From Hoppe-Seyler's "Zeitschrift über physiologische Chemie." gators, and the innumerable details which they accumilate in their respective branches of science, preclude the possibility of a modern "admirable Crichton." Werner's sigh, "True I know much, but yet I would know all," has been long acknowledged as an aspiration incapable of fulfilment, even supposing him to limit his desire of knowledge within the bounds of what is already known. To know "something of everything, and everything of something," is all that can be hoped for; day by day each science advances with such rapid strides, that one brain is incapable of grasping more than the general principles of one science; and any man who aims at enlarging the domain of science by fresh discovery, must content himself with confining his attention to a small corner, and by patient industry and indomitable perseverance seek to elicit some new facts.

Such, expressed in general terms, is the drift of the preface to Hoppe-Seyler's "Zeitschrift für physiologische Chemie." It seldom happens-unfortunately too seldom in this country-that medical men have more than a smattering of chemistry. A very low standard of chemistry is required for a medical degree, comprising a superficial knowledge of inorganic chemistry, chiefly of the non-metals; the merest smattering of organic chemistry, and ability sufficient to detect the acid and base in simple salts-such are the qualifications in chemistry necessary. for graduation in medicine. When the student, following out the prescribed course, comes to attend lectures on physiology, and hears-almost always for the first time-the names of the various principles contained in the animal fluids, in the brain, in the liver, and in the muscular tissue, they fail to convey any definite idea to bis mind, and he is utterly unable to comprehend, or even to form an idea of the reactions which take place in the animal organism. In Germany this state of things has been recognised in many universities, and special professors of physiological or animal chemistry have been appointed; these professors are not merely teachers, but are engaged in the active extension of their branch of science; and it is to facilitate the interchange: of ideas between them, and to afford a medium in which the results of their investigations may be brought together, that Hoppe-Seyler has undertaken the editorship of this journal.

One noticeable feature of the investigations of German physiological chemists at present, is the attention devoted to ascertaining the changes. which food undergoes in passing through the system. At least six memoirs on the subject are to be found in the nine published numbers ot the journal, comprising the work of a year and a half. The methods and results of these experiments are worthy of a short description.

The food which we eat consists for the most part of carbon, hydrogen, and nitrogen; all food, however, does not contain nitrogen; starch, sugar, and fat are devoid of that element. The carbon and hydrogen, after being absorbed by the tissues, and performing work, are cast off as waste material, partly by the lungs, in the form of carbonic acid and water-vapour, and also, to a much smaller extent, in the urine. An almost inappreciable amount of nitrogen escapes by the lungs; by far the largest portion passes into the urine in combination with bydrogen and carbon, in the form of urea-a white crystalline body. Now this substance, urea, possesses a chemical, as well as a physiological interest. It was formerly supposed that all chemical compounds could be divided into two grand classes: inorganic bodies, such as could be prepared from purely mineral matter; and organic bodies, those existing only in a living organism, or obtained from these compounds by a process of decomposition. It was, therefore, imagined that an insurmountable barrier separated the two classes. Urea was ranked as an organic substance, for it had never been obtained except from the organism, till Prof. Wöhler, of 